\title{
La théologie et la politique du Saint-Siège devant la Révolution française, 1789-1799, Gérard Pelletier
}

\section{Rita Hermon-Belot}

\section{(2) OpenEdition \\ 1 Journals}

\section{Édition électronique}

URL : https://journals.openedition.org/ahrf/2953

DOI : 10.4000/ahrf.2953

ISSN : 1952-403X

Éditeur :

Armand Colin, Société des études robespierristes

\section{Édition imprimée}

Date de publication : 1 mars 2002

Pagination : 119-127

ISSN : 0003-4436

\section{Référence électronique}

Rita Hermon-Belot, « La théologie et la politique du Saint-Siège devant la Révolution française,

1789-1799, Gérard Pelletier », Annales historiques de la Révolution française [En ligne], 327 | janvier-mars 2002, mis en ligne le 19 mars 2008, consulté le 22 avril 2022. URL : http://journals.openedition.org/ ahrf/2953 ; DOl : https://doi.org/10.4000/ahrf.2953 


\title{
LA THÉOLOGIE ET LA POLITIQUE DU SAINT-SIÈGE DEVANT LA RÉVOLUTION FRANÇAISE, 1789-1799
}

\author{
GÉRARD PELLETIER
}

M. Gérard Pelletier soutenait le 27 avril 2001, au Centre Malesherbes de Paris, sa thèse, La Théologie et la Politique du Saint-Siège devant la Révolution française, 1789-1799, pour le doctorat conjoint de l'Université Paris IVSorbonne et de l'Institut catholique de Paris, en vue de l'obtention du titre de docteur en histoire des religions - anthropologie religieuse et théologie. Le jury se composait de MM. les professeurs Jean-Marie Mayeur, rapporteur pour Paris IV, Philippe Boutry, lecteur pour Paris IV, Luigi Fiorani, lecteur pour Paris IV, Claude Bressolette, rapporteur pour l'Institut catholique, et Hervé Legrand, lecteur pour l'Institut catholique.

Le doctorant s'était donné pour principal objectif de retracer la manière dont les contemporains de la Révolution ont perçu l'événement, exercice qui s'est avéré tout autant d'histoire et de théologie, s'agissant notamment de saisir le regard de l'Église sur elle-même. On retrouvait là la question, soulevée par Bernard Plongeron, de l'indétermination régnant au sein de la congrégation vaticane chargée de suivre les événements révolutionnaires. Il fallait arriver à comprendre les choix de Pie VI, son refus à la fois des Droits de l'homme et de la Constitution civile du clergé, le retard mis à déclarer ce double refus et enfin sa perception de la Terreur. Un tel programme a d'abord nécessité un inventaire des archives romaines dans l'espoir de retrouver les archives constituées de la Congrégation des Affaires de France. Ont été étudiés les fonds de la Secrétairerie d'État, l'Archivio Secreto vaticano et la correspondance de la Propaganda fide, tandis que, malgré les destructions manifestes pour la période révolutionnaire, les archives du Saint-Office livraient des informations précieuses dans le dossier du cardinal Valenti Gonzaga, l'ensemble mettant en évidence la concomitance des actes de la curie autour du synode de Pistoia et des "Affaires de France ", et enfin la correspondance diplomatique. Les archives constituées de la Congrégation de France restant introuvables, il a fallu tenter une reconstruction à partir de morceaux épars.

L'étude visait d'abord à cerner l'orientation théologique de Pie VI et de la curie. Dans les histoires de la théologie, la période révolutionnaire reste souvent entre parenthèses alors que l'accent est mis sur l'unité de pensée d'une école romaine dont le moteur est l'anti-jansénisme et le refus des réformismes ecclésiastiques dans la seconde moitié du XVIIIe siècle. Pie VI prend son temps, demande conseil, mais en même temps agit en moderne au sens où il tente d'influer sur l'opinion publique de ses états. 
En totale adhésion avec les trois points de méthode définis par D. Julia, relire les textes, sortir de l'Hexagone et revenir aux orientations théologiques, le doctorant a voulu éclairer la volonté théologique à l'œuvre dans l'exercice de l'autorité pontificale de Pie VI, qui fait du pape la source unique de juridiction épiscopale sur un territoire ou un domaine de discipline. Mais il faut compter également avec les divergences de vue au sein de l'épiscopat en exil, visibles notamment au moment où on demande aux évêques leur démission au lendemain du Concordat. Malgré tous les efforts d'un Gerdil, se lit ici une exaspération déterminante pour le siècle à venir, étape irréversible même si on est encore bien loin de Vatican I. La grille de lecture essentiellement anti-janséniste de la Révolution bloque toute réflexion de fond sur le statut de la liberté de l'homme comme sur celui de l'homme dans la cité, d'autant plus que les premières avancées en ce domaine ont précisément été le fait de jansénistes ou de réformés. Le poids de la distance traditionnelle entre l'Église gallicane et la curie romaine s'avère donc extrêmement lourd. Mais si Rome appelle de ses vœux la Contre-Révolution, c'est exclusivement dans le courrier diplomatique et sans vouloir se lier avec les émigrés ni avec les Vendéens. Pie VI ne s'adresse pas à Louis XVIII, il joue toujours sur les différents plans, zelanti ou communicanti. On constate ici la fragilisation due à l'absence de toute réflexion de fond devant les interventions répétées du pouvoir civil dans les affaires ecclésiastiques au cours du XVIIIe siècle. À propos du Saint-siège luimême s'affirme le monolithisme des positions, face au bloc janséniste qu'elle voit dans la Révolution, Rome fait bloc à son tour.

Du point de vue de la théologie, cette étude est davantage théologie positive que spéculative. On suit ici, en référence aux travaux du père Congar, le rapport fécond entre théologie et histoire, avec ce risque inhérent, pour un dossier qui mène en droite ligne aux conciles de Vatican I et II, de sortir de la période. Le travail n'est pas construit en deux parties, mais organisé autour d'une troisième partie plus spécifiquement théologique placée au centre de la thèse. On compte également une grande absente, l'Église constitutionnelle, absence correspondant à la place que Rome voulut bien lui accorder, pratiquement nulle.

Certaines questions mériteraient d'être reprises, celle de la détermination systématique selon des canons «jansénistes» aux yeux de Rome, mais aussi celle que pose un Grégoire amoureux de Port-Royal au sortir de la Révolution, rejoignant les questions formulées par Timothy Tackett, Dale Van Kley et Catherine Maire. Litinéraire des évêques de France à la fin de l'Ancien Régime en fonction de leur choix durant l'exil et de la date de leur émigration exigerait un suivi personnel pour chacun d'entre eux. Il faudrait aussi définir en termes théologiques l'évolution des choix de l'Église catholique entre Quod Aliquantum et le concile Vatican I. Alors que, du côté des fidèles, des analyses particulières de situations pastorales mettraient 
certainement au jour une grande diversité de modalités pour d'authentiques attitudes chrétiennes.

Les membres du jury ont tous tenu à dire d'emblée la qualité de cette thèse dont ils espèrent la publication prochaine, à commencer par les deux codirecteurs. Mgr Claude Bressolette se réjouit non seulement pour le candidat, mais à la fois pour l'Université et pour l'Église de l'ampleur remarquable de cette recherche et le Pr Jean-Marie Mayeur salue un très beau travail, admirablement présenté. En adressant ses félicitations au chercheur qui s'est révélé ici, le père Hervé Legrand exprime son admiration pour la capacité de travail mais aussi pour la nuance de l'analyse. Le Pr Philippe Boutry évoque la genèse de la recherche en expliquant que le doctorant avait commencé à s'intéresser à la question en théologien plus qu'en historien et en rappelant sa participation au colloque de Valence qui commémorait la mort d'un pape sur le sol français. Le Pr Fiorani veut saluer la générosité intellectuelle qui consiste à s'aventurer dans un thème pour lequel on ne trouve aucune velléité de perspective théologique dans toute l'historiographie des $\mathrm{XIX}^{\mathrm{e}}$ et $\mathrm{XX}$ e siècles. Lacune de l'historiographie que le jury a unanimement rappelée en revenant toujours au nom de Mathiez, dont le Pr Boutry explique que le livre, Rome et le clergé français sous la Constituante, resté curieusement isolé et intangible, est bien sûr à l'orée du sujet. Il reposait pourtant sur un choix documentaire limité dans la mesure où il n'abordait pas les archives romaines. En revanche un poids considérable était attribué à l'affaire d'Avignon, ce qui devait constituer une doxa obligée que l'on retrouvait jusque dans les manuels d'histoire. C'est donc un renouvellement en profondeur qu'a opéré le doctorant. Le Pr Bressolette loue lui aussi les corrections apportées aux appréciations d'un Mathiez et même à celles d'un Bernard Plongeron. Il s'est agi pour le Pr Fiorani d'un véritable oubli historique pour une question restée en marge de la réflexion sur la Révolution française, le combat étant réduit aux dimensions d'un combat entre deux pouvoirs souverains sans prise en compte de la dimension de transcendance de l'Église, alors que l'événement révolutionnaire poussait celle-ci à se tourner vers son intériorité, ses propres dogmes et règlements disciplinaires.

L'ouverture des sources accomplie par G. Pelletier a été saluée par un jury unanime. Luigi Fiorani, lui-même professeur à l'Université mais aussi archiviste des Archives vaticanes, comme le Pr Boutry, voient là une véritable révolution historiographique à laquelle est associée une bibliographie riche de 1500 titres. Le Pr Brossolète assure que l'immensité formidable de ce travail le rendra absolument incontournable. L'intérêt du troisième volume, dont le Pr Boutry souhaite une publication séparée, a été vivement souligné : il s'agit d'un recueil des principaux brefs et parfois aussi allocutions consistoriales, donnés dans la forme utilisée à l'époque. Enfin l'ouvrage apporte une prosopographie des soixante-quinze cardinaux cités. Le 
Pr Fiorani insiste sur le fait qu'il ne s'est pas simplement agi ici de constituer un petit dictionnaire biographique mais de donner une véritable idée du milieu humain, de la sensibilité religieuse et de la carrière des hommes qui entourent le pape, ce qui fait apparaître aussi la faiblesse de Pie VI, son besoin constant d'être conseillé et soutenu. Un tel ensemble documentaire constitue, souligne le Pr Boutry, un instrument de travail de premier ordre.

Le jury a aussi été très sensible à la qualité de la forme. Le Pr Mayeur loue les proportions judicieuses d'un texte proprement dit de 538 pages, l'équilibre entre le texte et les notes, longues mais bien articulées à l'exposé et la qualité d'écriture de l'ensemble, dans un style clair, lumineux, précis, dense, vivant, notamment pour les péripéties très bien campées de l'histoire politico-militaire, témoignant de plus d'un véritable sens de l'humour. Le Pr Boutry apprécie la courtoisie de la discussion avec les prédécesseurs et le parti de donner les citations du texte original en latin et en français. Le Pr Mayeur salue l'effort de compréhension des différents camps et courants selon les préoccupations des contemporains et le sens de la mesure dont ce travail ne se départit jamais. La perspective adoptée a paru particulièrement pertinente et novatrice, une alliance entre histoire et théologie parfaitement résumée dans la formule citée par le Pr Mayeur: «essayer de penser ensemble l'historique et le théologique ». Les apports considérables de ce travail ont été longuement détaillés. Le Pr Legrand évoque le renouvellement de la connaissance de l'entourage et de la figure de Pie VI, par lequel cet ouvrage tient comme il est rare le propos de son titre. Une figure, avait précisé le Pr Bressolette, dont la vie a donné le cadre de périodisation, ce qui assure l'unité des problématiques théologiques. Le tableau de la «citadelle assiégée » apporte, indique le Pr Mayeur, à la fois une étude de sociologie du personnel de la curie, de ses modes de fonctionnements, notamment en ce qui concerne les nominations à des congrégations particulières, et un minutieux examen des Affaires de France, lesquelles peuvent mobiliser de une à trois congrégations fonctionnant parallèlement à celle qui se consacre à Pistoia; les travaux concernant le clergé et la Constitution civile du clergé avançant avec une extrême lenteur. Le Pr Boutry souligne aussi la qualité de l'analyse globale du personnel de la curie dans une approche qui se veut plus culturaliste que sociologique, ainsi que celle des passages remarquables consacrés à Pastoralis, au rôle de Mgr Fiorani et au serment romain. L'ouvrage présente ainsi un intérêt considérable pour l'étude d'un XIXe siècle dont il dessine l'héritage théologique et ecclésiologique, particulièrement autour de deux figures privilégiées excellemment choisies, Mgr Cappellari, futur Grégoire XVI, et Cesare Brandacoro, qui prononça l'éloge funèbre de Pie VI.

Le Pr Mayeur relève l'originalité de certains points précis parfois quelque peu éloignés de la théologie, un très intéressant chapitre consacré la diplomatie pontificale. Mais le traitement de la question des origines 
théologiques de la rupture montre à quel point l'affaire reste essentiellement romaine, la question en jeu étant celle de la primauté juridique face au courant réformiste dans une chronologie qui commence avant la Révolution et dans laquelle les événements de France ne sont qu'un élément des enjeux d'ensemble de la papauté. Le Pr Boutry constate à quel point l'Église constitutionnelle constitue le point aveugle assumé d'un Pie VI qui se refuse à en dire un seul mot. Mais la thèse apporte une nouvelle interprétation de ce silence en le mettant en rapport avec celui de l'épiscopat et avec les hésitations du roi. L'idée sous-jacente est celle que les jeux étant faits, il n'y a plus aucune raison de se presser.

L'un des acquis essentiels du travail, reconnu par tous les membres du jury, consiste à montrer à quel point la position romaine, notamment avec la prise en compte de la Constitution civile du clergé au moment précis où l'on s'attaque à Pistoia, est déterminée par l'anti-jansénisme qui domine à Rome. Pour le Pr Mayeur la manière dont la question est prise dans le prisme anti-janséniste la situe dans une histoire qui mène sans rupture à la papauté intransigeante du XIXe siècle. Un anti-jansénisme comme cadre d'analyse et mode d'appréhension déterminant dont le Pr Boutry retient qu'il permet de replacer la Révolution française dans une continuité, notamment autour du Giornale ecclesiastico, continuité qui précède le tournant intransigeant; ce qui atténue aussi considérablement le caractère d'exceptionnalité de la Révolution française aux yeux de Rome. Pareille vision constitue à la fois une faiblesse, dans la sous-estimation de la radicalité et de l'accélération du processus, mais aussi une force. Rome garde ses traditions séculaires. Depuis la primitive Église et ses persécutions et martyrs, elle s'inscrit dans une perspective qui se situe à une tout autre échelle que celle de l'événement. Le Pr Fiorani trouve dans l'étude de la genèse de Quod aliquantum et de Caritatis, d'importants éléments de réponse à ses interrogations sur l'unanimité que Pie VI avait pu trouver au sein de sa curie et des lumières sur l'École romaine. La cohérence entre le pape et la société ecclésiastique romaine est pour une large part assurée par la vigilance avec laquelle sont identifiées et frappées toutes les expressions intellectuelles et religieuses non alignées sur la position du pape. Létude apporte ainsi une réponse sans équivoque à la question de savoir si l'Église apporte des solutions nouvelles aux interrogations éveillées par les investissements du siècle.

Parmi les quelques réserves exprimées, aucune n'était de nature, selon l'expression du Pr Fiorani, à faire «couler le sang ». Lui-même remarque le point de vue très intellectuel selon lequel G. Pelletier aborde l'«École romaine ". Il faudrait aussi faire leur place aux écoles de théologie dont on ne sait rien pour l'époque révolutionnaire. La grande école des Pères Jésuites au Collège romain a été supprimée à la fin du siècle. Comment le monde scolaire, les livres, les élèves ont-ils évolué ? Des voix très diverses et multiples s'expriment notamment dans le Giornale ecclesiastico romano, 
terroir où la vraie Contre-Révolution prend ses racines, et pas seulement dans les milieux de la curie romaine. Le Pr Bressolette souhaiterait la suppression de la formule «Dieu par le sacrement». La juridiction selon la théologie romaine ne se référerait-elle plus tant à Dieu qu'au pape ou ne s'agit-il que de délimiter une juridiction fondée par le sacrement? Si la source du pouvoir d'ordre est le sacrement, quelle est celle du pouvoir de juridiction? Mais il reconnaît que l'objectif théologique de la thèse était bien de comprendre la source et le fondement de l'exercice de la primauté romaine (le terme de collège évoque cependant la tête et les membres).

Évoquant notamment l'élément sociologique présent dans la thèse d'État de Philippe Boutry, le Pr Legrand rappelle que les cardinaux de la curie constituaient aussi un corps social pris dans un réseau de bénéfices familiaux qui ne les prédisposait guère à comprendre la nouveauté, dimension que l'on pourrait considérer dans une histoire un peu plus «vraie » et pas seulement intellectuelle, en tenant compte du facteur que représente l'ascension de la noblesse provinciale aux dépens de la noblesse romaine. Il fait remarquer que le vere episcopalis dont il est question ici n'est pas repris des termes de la doctrine de Vatican I, le pape n'y est pas évêque de l'Église catholique mais appartenant à l'Église catholique. Les deux termes de la formule de la papauté monarchique exigent eux aussi explication. Il faut montrer que ce type d'identification explique la nota previa de Lumen Gentium, III, s'agissant d'une collégialité qui reste à la discrétion du pape. Enfin, Vatican I donne une définition en quatorze lignes de l'infaillibilité. La résumer en quatre est dogmatiquement dangereux par rapport à l'opinion commune. Le Pr Bressolette intervient ici pour préciser que G. Pelletier spécifie bien que l'infaillibilité est attachée au ministère du pape.

Les réponses du doctorant donnent lieu à une fructueuse discussion sur nombre de points centraux. Le Pr Bressolette estime que le chapitre V, montrant l'importance de la pratique de la primauté de juridiction, mériterait d'être développé davantage en prenant garde toutefois à ce que le terme de « gallican » a d'embarrassant à cause de la connotation liée à Vatican I. Si l'on se réfère aux travaux de Ratzinger, ce qui est déterminant en théologie, ce n'est pas la logique du discours mais les faits, les circonstances exceptionnelles dans lesquelles se révèle la primauté de juridiction du pape. D'où le très grand intérêt de l'épluchage de passages ambigus dans leur formulation, ainsi de cette interprétation politique de l'infaillibilité qui fait penser à l'argumentation de Joseph de Maistre ou de la vision de la liberté de conscience comme « délire », conception qui découle de la doctrine de l'indifférentisme au sein d'une Église inquiète de la responsabilité des princes auxquels il incombe de maintenir les populations dans la vraie religion. Sur ce terme de « délire », G. Pelletier répond que Grégoire XVI reprend le terme à Pie VI. S'agit-il d'une expression plus ancienne ou apparaît-elle avec la Révolution? 
Le Pr Fiorani a trouvé dans le portrait de Bolgeni un auteur de pensées contre-révolutionnaires capable de se ranger à la nécessité de prêter le serment romain à la république. N'est-ce que par opportunisme? Par rapport à l'opinion reçue selon laquelle il ne saurait y avoir de soutien du milieu ecclésiastique romain que par opportunisme, il ne faut pas négliger une réelle sympathie pour la dimension populaire de la Révolution. De ce point de vue, la place consacrée à Consalvi est-elle suffisante ? Il faudrait également donner la parole aux chrétiens orthodoxes modérément favorables à la Révolution, en référence notamment aux travaux de l'historien Vittorio Emmanuele Giuntella évoquant un catholicisme à caractère fortement social. En ce qui concerne Bolgeni, G. Pelletier reconnaît bien volontiers que la thèse reste à faire. Est-il le véritable auteur de Pastoralis? S'il n'est pas vraiment un novateur en théologie, il est effectivement beaucoup plus libre en politique. Ses positions sur le serment ont d'ailleurs été désavouées. Ainsi de féroces anti-jansénistes pourraient-ils avoir une certaine forme de liberté politique ? Quant au courant qui se veut à la fois orthodoxe sur le plan religieux et démocrate en politique, il y a effectivement des hommes libres autour de la curie romaine, parmi les cardinaux, un Consalvi qui fait à ce moment ses classes politiques. Pour ce qui est enfin de la diversité des écoles, il est certain que le travail reste à faire, notamment à partir de la correspondance de Gerdil qui prend les choses en main, fixant chaque année les thèmes du cours de théologie du Collège romain.

Le Pr Fiorani rappelle que le bref Pastoralis sollicitudo est un des documents les plus tourmentés du pontificat de Pie VI sur lequel ont même couru des rumeurs de faux. L'authenticité du bref ne fait pourtant aucun doute. En revanche, s'il était facile d'affirmer le devoir d'obéissance des catholiques envers la monarchie, il en allait bien autrement aux yeux de Rome à l'égard du Nouveau Régime. G. Pelletier reconnaît que la formule «rien de nouveau » à propos de Pastoralis sollicitudo était certainement trop rapide, mais on ne trouve dans le bref qu'une construction sur Romains XIII. C'est parce qu'allant au but en vingt lignes, il n'est pas rédigé dans les formes classiques que le document a pu être accusé de faux. Il fait cependant preuve d'un effort de mansuétude à l'égard du Directoire en tant que gouvernement qui permet de sortir du chaos. L'occasion diplomatique est manquée à cause de l'entêtement du Directoire à obtenir le désaveu des brefs de 1791, mais le bref est aussi une manière de refus, la liberté y reste un délire.

Le Pr Legrand pose la question du Tiers-parti exhumé par la thèse d'Émile Appolis et qui représente une ligne de recherche pertinente non explorée. Mais parmi les jansénistes ou les zelanti, on ne compte guère de catholiques éclairés qui soient également bons théologiens. Pie VI d'ailleurs résiste aux zelanti. L'histoire a-t-elle vraiment laminé le Tiers-parti? G. Pelletier remarque qu'Appolis, dans son étude, s'arrête avant cette période. De fait, le Tiers-parti ne fait pas l'unanimité. L'existence d'hommes 
ayant eu cette liberté de pensée n'est pas douteuse, mais celle d'un parti? Pie VI rogne les extrêmes pour ne laisser s'exprimer que les voix de Gerdil et d'Antonelli. Tous d'ailleurs, à l'instar même d'un Borgia ou d'un Spedalieri, finissent généralement par se retrouver dans la mobilisation anti-janséniste. Le Pr Legrand félicite aussi le doctorant de ne pas avoir écrit l'histoire de Pie VI à partir de son dénouement. La figure s'en trouve revalorisée, notamment dans ses qualités de prudence, notion dont il faudrait toutefois pouvoir déterminer la juste perception. Ainsi du passage dans lequel le pontife se désigne lui-même comme "placé par la divine prudence». Un tel énoncé constitue-t-il une invitation à suspendre le jugement? Il faut en effet, estime G. Pelletier, une forme de suspension pour opérer le travail. L'Encyclique porte un regard réaliste sur la société de son temps, mais l'absence de toute capacité d'anticipation aboutit infailliblement à la condamnation de tout réformisme. Ce pape béatifie aussi un quart de femmes, ce qui est indiscutablement nouveau, mais ces quelques transactions ne sont qu'empiriques dans une aspiration qui ne tend qu'à la conservation. Et si l'on note une volonté de réforme à l'œuvre dans les États, les réactions de la population et de la noblesse y font massivement obstacle. L'une des grandes difficultés de l'interprétation tient au fait que le pape n'a ni carnet de bord ni notes personnelles. On ne dispose que de quelques notes de secrétaires, plutôt frileuses. Très vite aussi, il n'est plus temps de tenter des avancées sur un terrain devenu trop dangereux. Lors du voyage à Vienne que le pape avait été le premier à oser dans les temps contemporains, la rencontre avec Joseph II s'était déjà achevée sur un véritable traumatisme.

Après avoir convenu avec le doctorant de l'étroitesse du point de vue romain, le Pr Legrand aborde en dernier point l'insistance des conservateurs à désigner les autres comme innovateurs, position qui constitue en ellemême une innovation. L'adjectif «magistral » lorsqu'il s'agit du Triunfo de la Santa sede doit être nuancé. Encore un triomphe comme celui-là, faisait remarquer M.-J. Congar, et le désastre est irrémédiable. G. Pelletier explique que le caractère de nouveauté tenait justement au fait que cet ouvrage était construit comme un cours magistral. Il a aussi repris le terme d'« infaillible » dans un sens effectivement différent de celui que lui a donné Vatican I. Quant à la notion de «conservatisme » comme innovation, c'est là en effet la fine pointe qu'il a voulu donner à son travail.

«Qu'est-ce qu'une très bonne thèse? » s'interroge en manière de conclusion le Pr Boutry. Louvrage doit réaliser un véritable apport au plan documentaire, comme cela a été amplement salué ici, un apport au plan méthodologique, G. Pelletier y satisfait remarquablement en remettant les logiques théologiques au cœur de tous les autres aspects, un apport problématique, le jansénisme comme grille de lecture universelle, et enfin un «je ne sais quoi» dont ce travail fait incontestablement preuve. Et si l'on a 
entendu quelques petites réserves, on remarquera qu'elles ont été davantage le fait des théologiens que des historiens. Affaire de tempérament?

Après une courte délibération, le jury décerne le titre de docteur en Histoire des religions - anthropologie religieuse et théologie à M. Gérard Pelletier, avec la mention «très honorable » et ses félicitations à l'unanimité.

Compte rendu de Rita HERMON-BELOT 